\title{
Prostate cancer screening practices of African- American and non-African-American US primary care physicians: a cross-sectional survey
}

This article was published in the following Dove Press journal:

International Journal of General Medicine

18 September 2012

Number of times this article has been viewed

\section{Thomas B Richards' \\ Sun Hee Rim' \\ Ingrid J Hall' \\ Lisa C Richardson' \\ Louie E Ross²}

'Division of Cancer Prevention and Control, National Center for Chronic Disease Prevention and Health Promotion, Centers for Disease Control and Prevention, Atlanta, GA, USA; ${ }^{2}$ North Carolina A and T State University, Greensboro, NC, USA
Correspondence: Thomas B Richards Epidemiology and Applied Research Branch, Division of Cancer Prevention and Control, Centers for Disease Control and Prevention, 4770 Buford Highway NE (K-55), Atlanta,

GA 3034I-37I7 USA

Tel + I 7704883220

Fax +I 7704884639

Email trichards@cdc.gov
Purpose: We explored whether African-American (AA) primary care physicians (PCPs) have different prostate cancer screening practices compared to non-AA PCPs, after adjustment for potential confounding factors such as the proportion of AA patients in PCP practices.

Methods: We used SAS/SUDAAN to compare weighted responses from AA PCPs $(n=604)$ with those from non-AA PCPs $(n=647)$ in the 2007-2008 National Survey of Primary Care Physician Practices Regarding Prostate Cancer Screening. We used multivariate logistic regression to calculate the weighted odds ratios (OR) and $95 \%$ confidence intervals (CI).

Results: We found that AA PCPs had higher odds of working in practices with above-the-median ( $\geq 21 \%$ ) proportions of AA male patients (OR, 9.02; 95\% CI: 5.85-13.91). A higher proportion of AA PCPs $(53.5 \%$; 95\% CI: 49.5-57.4) reported an above-the-median proportion ( $\geq 91 \%)$ of PSA testing during health maintenance exams as compared to non-AA PCPs $(39.4 \%$; $95 \%$ CI: $35.5-43.4 ; P<0.0002)$. After adjusting for the proportion of AA patients and other factors, we found that AA PCPs had higher odds of using PSA tests to screen men (OR, 1.74; 95\% CI: 1.11-2.73).

Conclusion: This study quantifies the magnitude of the differences reported in previous focus group studies. Our results may be helpful in hypothesis generation and in planning future research studies.

Keywords: African-American, physician practice patterns, prostate-specific antigen, screening tests

\section{Introduction}

Prostate cancer screening practices by African-American (AA) primary care physicians (PCPs) comprise an important area of primary care research for several reasons. First, incidence and mortality rates for prostate cancer are higher in African-American (AA) men than in other racial/ethnic groups. ${ }^{1}$ Second, patients from specific race and ethnic groups, including AA patients, may preferentially seek care from PCPs of their own race. ${ }^{2-4}$ Finally, some AA PCPs have relatively large proportions of AA patients in their practices..$^{5-7}$

AA PCPs account for approximately 5\% of all PCPs in the United States. ${ }^{8}$ If respondents for a survey were selected from lists with no information regarding PCP race, the study may not include sufficient numbers of AA PCPs to provide accurate estimates for that group. Therefore, in order to accurately describe PCP practice patterns, knowledge, and beliefs in the area of prostate cancer screening, we conducted a nationally representative survey that included a sufficient number of AA and non-AA PCPs to enable accurate estimates for both groups. ${ }^{8}$ 
In this study, we investigate whether AA PCPs differ from non-AA PCPs in their prostate cancer screening practices after adjustment for potential confounding factors such as the proportion of AA patients seen in different practices.

\section{Methods}

For the current study, we analyzed the 2007-2008 National Survey of Primary Care Physician Practices Regarding Prostate Cancer Screening, which was conducted by the Centers for Disease Control and Prevention (CDC). ${ }^{8}$ This mailed survey presented PCPs with questions regarding their individual practices and characteristics, their prostate cancer screening practices, and other factors that may influence screening. The Institutional Review Board at CDC and the Office of Management and Budget reviewed and approved this survey.

The survey methods have been described previously. ${ }^{8,9}$ Briefly, respondent PCPs were required to be office-based physicians in family practice, general practice, or general internal medicine, work at least 8 hours per week in outpatient care, and provide routine health maintenance exams (HMEs) at their site of primary practice care. Respondent PCPs were excluded if their practice included no male patients over the age of 40 years. The survey sample frame was taken from the American Medical Association Physician Masterfile. Disproportionate stratified random sampling was used to provide a sufficient number of AA PCPs for comparisons with non-AA PCPs. Data collection began in September 2007 and was completed in October 2008. Respondents self-identified their race on the survey by checking all that applied among the following options: white, black or African-American, Asian, Native Hawaiian or other Pacific Islander, and Native American or Alaska Native. We defined AA PCPs using the black or AA category, and non-AA PCPs as including all other categories. The sample frame listed 31 AA PCPs who identified themselves as non-AA on the questionnaire and 12 non-AA PCPs who identified themselves as AA on the questionnaire. In our analysis, we used race information reported by the PCP on the questionnaire if different from the information on the sample frame. The final number of eligible participants was 1,100 AA PCPs and 1,119 non-AA PCPs. The overall survey response rate was $54.9 \%(604 / 1,100)$ for AA PCPs and $57.8 \%(647 / 1,119)$ for non-AA PCPs.

The survey questions had a variety of formats, including instructions to mark "yes" or "no," fill in a blank space with a percentage, or indicate one of several options on a Likert scale (eg, strongly disagree, disagree, neither agree nor disagree, agree, or strongly agree). We converted responses for all questions into two categories in order to simplify the analysis and interpretation of results. For continuous variables, we used the median to construct the two categories (ie, $<$ the median percentage vs $\geq$ the median percentage). For Likert scale questions, we formed two groups by coding "agree" and "strongly agree" as one category (ie, "agree") and coding "strongly disagree", "disagree", and "neither agree nor disagree" as the second category (ie, "other responses").

To evaluate whether AA PCPs and non-AA PCPs had similar prostate cancer screening practices after adjustment for potential confounding factors such as the proportion of AA patients seen in different practices, we focused on questions with $\geq 10 \%$ differences in responses between AA and non-AA PCPs. This subset helped reduce the likelihood of false positives that would be observed by random chance when evaluating multiple questions.

Our conceptual model viewed prostate cancer screening practices as being influenced by multiple factors, including physician characteristics (PCP sex and race), type of organization and location (single specialty group practice, practice location in the South, and urban, inner city practice location), patient characteristics ( $\geq 26 \%$ of patients were Medicare-eligible, $\geq 41 \%$ of patients were male, and $\geq 21 \%$ of patients were AA), prostate cancer screening clinical practices ( $\geq 91 \%$ of HME patients receive prostate-specific antigen [PSA]-based screening, and physicians provided patients with educational materials), and other factors that may influence screening (PCPs believe that digital rectal exams [DREs] are a reliable tool for cancer detection, and that PSA tests help protect against medical malpractice claims, PCPs report that most medical colleagues provide DREs and indicate that patients provide family history of prostate cancer).

We used SAS software (version 9.2; SAS Institute, Inc, Cary, NC) with callable SUDAAN version 10.0 (RTI International, Research Triangle, NC) for all data analysis, adjusting for the survey design, stratification, and weights. We calculated survey-weighted proportions, $95 \%$ confidence intervals (CI), and chi-square $P$-values for each variable in the conceptual model. We used survey-weighted logistic regression to calculate unadjusted odds ratios (OR) and 95\% CIs for responses from AA PCPs, with non-AA PCPs as the reference group, for each of the other variables in the conceptual model, one variable at a time. We used multivariate survey-weighted logistic regression to calculate adjusted ORs for responses from AA PCPs, using non-AA PCPs as the reference group, where the independent variables included all other variables in the conceptual model. 


\section{Results}

After adjustment for survey design, stratification, and sample weights, we found that 4.8\% (95\% CI: 4.6-5.0) of all respondents were AA and 95.2\% (95\% CI: 95.0-95.4) were non-AA PCPs. The respondents represented 4,544 AA and 89,995 non-AA PCPs nationally. More than $80 \%$ of the respondent PCPs were board certified, regardless of race. Differences in AA and non-AA PCP responses were $<10 \%$ for $85.5 \%(174 / 206)$ of the questions.

The percentages, 95\% CIs, and ORs in Table 1 are adjusted for the survey design, stratification, and weights, but are not adjusted for confounders. Compared with non-AA PCPs, a larger proportion of AA PCPs were women, had an office in the South, and were in an urban, innercity location. AA PCPs reported smaller proportions than non-AA PCPs of practicing in a single-speciality group practice, male patients and of patients using Medicare as their primary payment method, but larger proportions of AA patients than non-AA PCPs.

AA PCPs also reported that a larger proportion (above the median of $91 \%$ ) of healthy men received PSA testing during their health maintenance exams (Table 1). Although proportions were relatively low for both AA and non-AA PCPs, we found that AA PCPs were more likely to provide educational materials informing men about prostate cancer screening.

Survey results additionally showed that AA PCPs were more likely than non-AA PCPs to agree that the DRE was a reliable tool for cancer detection in average-risk men (Table 1). In contrast, AA PCPs were less likely than non-AA PCPs to agree that PSA testing of average-risk men helped protect the PCP against malpractice claims, that male patients reported information about their family history of prostate

Table I Unadjusted survey weighted percents, 95\% confidence intervals ( $\mathrm{Cl}$ ), and odds ratios (ORs) for responses from AA and nonAA PCPs

\begin{tabular}{|c|c|c|c|c|c|}
\hline Characteristic & PCP & $\%$ & $95 \% \mathrm{Cl}$ & OR & $95 \% \mathrm{Cl}$ \\
\hline \multicolumn{6}{|l|}{ Physician characteristics } \\
\hline \multirow[t]{2}{*}{ Female } & AA & 47.6 & $43.9-51.4$ & 2.31 & $1.84-2.90$ \\
\hline & non-AA & 28.3 & $24.9-31.9$ & 1.0 & - \\
\hline \multicolumn{6}{|l|}{ Type of organization and location } \\
\hline \multirow[t]{2}{*}{ Single-specialty group practice } & AA & 28.5 & $25.3-32.1$ & 0.55 & $0.43-0.69$ \\
\hline & non-AA & 42.3 & $38.4-46.2$ & 1.0 & - \\
\hline \multirow[t]{2}{*}{ Practice location in South } & AA & 60.1 & $56.3-63.8$ & 3.13 & $2.49-3.95$ \\
\hline & non-AA & 32.5 & $28.8-36.3$ & 1.0 & - \\
\hline \multirow[t]{2}{*}{ Urban, inner city } & AA & 30.9 & $27.5-34.6$ & 3.74 & $2.75-5.09$ \\
\hline & non-AA & 10.7 & $8.5-13.4$ & 1.0 & - \\
\hline \multicolumn{6}{|l|}{ Patient characteristics } \\
\hline \multirow[t]{2}{*}{ Medicare $\geq 26 \%$ of patients } & AA & 37.7 & $34.0-41.5$ & 0.58 & $0.46-0.73$ \\
\hline & non-AA & 51.0 & $46.9-55.0$ & 1.0 & - \\
\hline \multirow[t]{2}{*}{ Male $\geq 41 \%$ of patients } & AA & 29.8 & $26.4-33.4$ & 0.45 & $0.36-0.56$ \\
\hline & non-AA & 48.7 & $44.8-52.6$ & 1.0 & - \\
\hline \multirow[t]{2}{*}{$A A \geq 21 \%$ of male patients } & $\mathrm{AA}$ & 76.4 & $73.1-79.5$ & 10.93 & $8.42-14.19$ \\
\hline & non-AA & 22.9 & $19.7-26.4$ & 1.0 & - \\
\hline \multicolumn{6}{|l|}{ Screening clinical practices } \\
\hline \multirow[t]{2}{*}{ HME patients receive PSA $\geq 91 \%$} & AA & 53.5 & $49.5-57.4$ & 1.77 & $1.4 \mathrm{I}-2.23$ \\
\hline & non-AA & 39.4 & $35.5-43.4$ & 1.0 & - \\
\hline \multirow[t]{2}{*}{ Provide educational materials ${ }^{\mathrm{b}}$} & AA & 27.2 & $24.0-30.7$ & 2.08 & $1.57-2.75$ \\
\hline & non-AA & 15.2 & $12.6-18.3$ & 1.0 & - \\
\hline \multicolumn{6}{|l|}{ Beliefs/factors influencing screening } \\
\hline \multirow[t]{2}{*}{ DRE is reliable tool for cancer detection ${ }^{c}$} & AA & 62.4 & $58.6-66.0$ & 1.52 & $1.22-1.90$ \\
\hline & non-AA & 52.1 & $48.1-56.0$ & 1.0 & - \\
\hline \multirow{2}{*}{ PSA helps protects from malpractice ${ }^{c}$} & AA & 58.8 & $55.0-62.6$ & 0.60 & $0.47-0.76$ \\
\hline & non-AA & 70.5 & $66.7-73.9$ & 1.0 & - \\
\hline \multirow[t]{2}{*}{ Men provide family history of prostate cancer ${ }^{b}$} & AA & 51.8 & $48.0-55.6$ & 0.61 & $0.49-0.77$ \\
\hline & non-AA & 63.7 & $59.8-67.5$ & 1.0 & - \\
\hline \multirow[t]{2}{*}{ Most medical colleagues provide DREs ${ }^{c}$} & AA & 56.8 & $53.0-60.6$ & 0.61 & $0.48-0.77$ \\
\hline & non-AA & 68.2 & $64.4-71.9$ & 1.0 & - \\
\hline
\end{tabular}

Notes: ${ }^{\mathrm{N}} \mathrm{N}=1$ 1,256. Percents, $95 \% \mathrm{Cls}$, and ORs are adjusted for the survey design, stratification, and weights, but are not adjusted for multiple variables. ORs for each characteristic are calculated with survey-weighted logistic regression and compare responses from AA PCPs with non-AA PCPs as the reference group. All comparisons have $P$-value $\leq 0.0002$; busually/always; cagree/strongly agree.

Abbreviations: AA, Black, African, or African American; Cl, confidence interval; DRE, digital rectal exam; HME, health maintenance exam; OR, odds ratio; PCP, primary care physician; PSA, prostate-specific antigen test; USPSTF, US Preventive Service Task Force. 
cancer, or that most of their medical colleagues routinely provided DREs to male patients.

In adjusted logistic regression analysis, AA PCPs had higher odds than non-AA PCPs of being female, practicing in the South, having an innercity urban location, and reporting a larger proportion of AA patients in their practices (Table 2). Compared to non-AA PCPs, the AA PCPs had lower odds of practicing in a single specialty group setting and of reporting larger proportions of Medicare or male patients in their practices. AA PCPs had higher odds of screening their health maintenance exam patients with PSA tests, providing educational materials to their patients about prostate cancer

Table 2 Adjusted odds ratios for responses from AA PCPs and non-AA PCPs ${ }^{\mathrm{a}}$

\begin{tabular}{|c|c|c|c|}
\hline Characteristic & $\mathbf{P C P}$ & OR & $95 \% \mathrm{Cl}$ \\
\hline \multicolumn{4}{|l|}{ Physician characteristics } \\
\hline \multirow[t]{2}{*}{ Female } & AA & 2.25 & $1.4 \mid-3.60$ \\
\hline & non-AA & 1.0 & - \\
\hline \multicolumn{4}{|l|}{ Type of organization and location } \\
\hline \multirow[t]{2}{*}{ Single specialty group practice } & $\mathrm{AA}$ & 0.44 & $0.27-0.72$ \\
\hline & non-AA & 1.0 & - \\
\hline \multirow[t]{2}{*}{ Practice location in South } & $\mathrm{AA}$ & 2.65 & $1.67-4.21$ \\
\hline & non-AA & 1.0 & - \\
\hline \multirow[t]{2}{*}{ Urban, inner-city } & AA & 3.23 & $1.90-5.50$ \\
\hline & non-AA & 1.0 & - \\
\hline \multicolumn{4}{|l|}{ Patient characteristics } \\
\hline \multirow[t]{2}{*}{ Medicare $\geq 26 \%$ of patients } & AA & 0.62 & $0.40-0.96$ \\
\hline & non-AA & 1.0 & - \\
\hline \multirow[t]{2}{*}{ Males $\geq 4 \mathrm{I} \%$ of patients } & $\mathrm{AA}$ & 0.28 & $0.17-0.43$ \\
\hline & non-AA & 1.0 & - \\
\hline \multirow[t]{2}{*}{$A A \geq 21 \%$ of male patients } & AA & 9.02 & $5.85-|3.9|$ \\
\hline & non-AA & 1.0 & 1.0 \\
\hline \multicolumn{4}{|l|}{ Screening clinical practices } \\
\hline \multirow[t]{2}{*}{ HME patients receive $\mathrm{PSA} \geq 91 \%$} & $\mathrm{AA}$ & 1.74 & I.II-2.73 \\
\hline & non-AA & 1.0 & - \\
\hline \multirow[t]{2}{*}{ Provide educational materials } & AA & 2.34 & $1.37-3.99$ \\
\hline & non-AA & 1.0 & - \\
\hline \multicolumn{4}{|l|}{ Beliefs/factors influencing screening } \\
\hline DRE is reliable tool for cancer & $\mathrm{AA}$ & 2.36 & $1.46-3.80$ \\
\hline detection & non-AA & 1.0 & - \\
\hline \multirow[t]{2}{*}{ PSA helps protects from malpractice } & AA & 0.43 & $0.27-0.68$ \\
\hline & non-AA & 1.0 & - \\
\hline Men provide family history & AA & 0.57 & $0.35-0.94$ \\
\hline of prostate cancer & non-AA & 1.0 & - \\
\hline Most medical colleagues provide & $\mathrm{AA}$ & 0.61 & $0.39-0.94$ \\
\hline DREs & non-AA & 1.0 & - \\
\hline
\end{tabular}

Notes: ${ }^{a}$ ORs were calculated using survey-weighted logistic regression, and are adjusted for the survey design, stratification, weights, and multiple variables. The ORs compare responses from AA PCPs with non-AA PCPs as the reference group and adjust for confounders by including all other characteristics listed in this table as independent variables. The Hosmer-Lemeshow Satterthwaite Goodness of Fit Test $P$-value for this model was 0.14 .

Abbreviations: AA, Black, African, or African American; $\mathrm{Cl}$, confidence interval; $\mathrm{DRE}$, digital rectal exam; HME, health maintenance exam; OR, odds ratio; $\mathrm{PCP}$, primary care physician; PSA, prostate-specific antigen test; USPSTF, US Preventive Service Task Force. screening, and believing DRE to be a reliable tool for cancer detection. AA PCPs had lower odds of reporting that PSA testing protected them from medical malpractice, their male patients provided a family history of prostate cancer, or that most of their medical colleagues provided DREs.

\section{Discussion}

Using a national survey with sufficient numbers of AA PCPs to enable accurate estimates for both AA and non-AA PCPs, we found that the magnitude of the difference between AA PCP and non-AA PCP responses was $<10 \%$ for most questions. However, we also identified a small number of questions with differences $\geq 10 \%$ in magnitude and $P$-values $\leq 0.0002$, values that persisted after adjustment for factors such as the proportion of AA patients in the PCP practices.

Prior to the current study, little quantitative data was available concerning differences in prostate cancer screening practices between AA and non-AA PCPs. Focus groups have been conducted, but involving relatively small numbers of PCPs. ${ }^{6,7}$ The focus group reports recommended additional research with larger numbers of PCPs in order to confirm the findings of their studies.

The current study results are generally consistent with those from previous focus group studies, ${ }^{6,7}$ but go beyond those studies by quantifying the magnitude of the differences and providing additional insights. For example, in the AA focus group study, most physicians indicated that they did not use any educational materials because the available materials were of poor quality and the viewpoints expressed were not appropriately balanced. ${ }^{7}$ In the current study, the proportion of AA PCPs providing patients with supplementary educational materials was relatively small, but the proportion of AA PCPs providing educational materials was higher than in non-AA PCPs.

In both the AA PCP focus group study ${ }^{7}$ and the current national survey, AA PCPs reported higher proportions of AA patients. These results are consistent with studies suggesting that patients from specific race and ethnic groups, including AA patients, may preferentially seek care from PCPs of their own race or reside in areas mostly served by AA PCPs. ${ }^{2-4} \mathrm{It}$ is important to note that AA physicians comprise an important source of healthcare for AA patients..$^{2-4}$

The 2007-2008 survey does not provide a definitive answer as to why AA PCPs reported more frequently than non-AA PCPs that their HME patients receive PSA tests. One hypothesis is that AA PCPs are more likely to screen AA patients in their practices because these physicians are more aware of the higher burden of prostate disease in 
AA patients. ${ }^{7}$ This greater awareness may have been obtained through either professional observation of patients or the personal experience of friends or family members diagnosed with prostate cancer. ${ }^{10}$ Some AA PCPs may also believe that the ratio of benefit to harm for screening is more favorable among AA men.?

During the duration of the current survey, the clinical considerations statement in US Preventive Services Task Force (USPSTF) recommendations included wording that may have permitted PCPs to come to conflicting conclusions regarding prostate cancer screening. ${ }^{11}$ The USPSTF summary recommendation stated that "the evidence is insufficient to recommend for or against routine screening" using PSA testing or DRE. ${ }^{11}$ In contrast, some PCPs may have concluded from this statement that PSA tests should not be performed, particularly because of the USPSTF clinical consideration statement that "screening is associated with important harms, including frequent false-positive results and unnecessary anxiety, biopsies, and potential complications of treatment of some cases." ${ }^{11}$ Another possibility is that some PCPs may have concluded that PSA testing was permissible, particularly because of the USPSTF clinical consideration statement that "if early detection improves health outcomes, the population most likely to benefit from screening will be men 50 to 70 years of age who are at average risk and men older than 45 years of age who are at increased risk (AfricanAmerican men and those with a first-degree relative with prostate cancer)." 11

The reported difference by PCP race in the frequency that their HME patients received PSA tests may have also been influenced by factors that were not addressed in the 2007-2008 questionnaire. For example, the questionnaire did not include information regarding PCP personal health practices, such as physical activity, diet, drinking habits, and smoking habits. ${ }^{10}$ In a different study of prostate cancer screening practices reported by US medical students, personal health practices confounded the association between the student's race and PSA screening frequency. ${ }^{10}$ In that study, no statistically significant relationship between the student's race and the frequency of offering PSA counseling was found after adjusting for the student's personal health practices. ${ }^{10}$

Most PCPs agreed or strongly agreed that providing PSA testing to average risk patients helped protect them from medical malpractice claims. PCP concern regarding legal liability for delay in diagnosis has been suggested as one of the potential factors contributing to the common use of PSA testing in the US. ${ }^{12}$ Additional research is needed to test this hypothesis. ${ }^{13} \mathrm{~A}$ study has reported on whether PCPs changed their prostate cancer screening behavior before, during, and after one of the PCPs in their community was sued for using shared decision making rather than PSA testing. ${ }^{13}$ This study concluded that PCPs in that community continued to use shared decision making and to let patients decide whether to be screened, but the use of PSA-testing increased..$^{13}$ In the current study, medical malpractice concerns appeared less influential for AA compared with non-AA PCPs. The reasons for this difference are not known.

The current study has several strengths. First, the questionnaire content was developed using a broad range of methodologies including literature reviews, PCP focus groups, and pilot testing of the survey instrument. ${ }^{5-7}$ Second, the study included relatively large numbers of AA PCPs, which enabled a more detailed examination of practice differences by PCP race. Third, questions with larger $(\geq 10 \%)$ differences had a chi-square $P$-value of $\leq 0.0002$, reducing the likelihood of false positives that may have been observed by random chance if multiple questions were evaluated statistically.

The current study also has limitations. First, although survey response rates were similar for AA PCPs and non-AA PCPs, more than $40 \%$ of PCPs in both groups were nonrespondents. These response rates are consistent with previous observations that physicians often may be less likely than other groups to respond to mail surveys. ${ }^{14}$ For the current survey, the prostate cancer screening practices of PCP nonrespondents is unknown. ${ }^{15}$ Second, we dichotomized variables in order to simplify the analysis and interpretation of results, and this simplification may have resulted in loss of some information from the original multiple categories. ${ }^{16}$ Third, the survey results were not validated by chart review or patient interviews. ${ }^{17}$

Fourth, from the perspective of current public health practice implications, an unknown is the extent to which current PCP practice patterns may be different from those in the 2007-2008 survey due to changes over time in PSA screening recommendations. ${ }^{11,18,19}$ For example, in 2012, the USPSTF recommended against performing PSA-based screening for prostate cancer in all men because there was "moderate or high certainty that the service has no net benefit or that the harms outweigh the benefits." ${ }^{19}$ The 2012 USPSTF recommendations for AA men were the same as those for non-AA men. ${ }^{19}$ These recommendations have been controversial. ${ }^{20,21}$ In addition to several other objections, critics argue that the USPSTF underestimated the benefits and overestimated the harms of PSA screening, particularly for higher risk populations such as AA men. ${ }^{20}$ An updated 
PCP survey is necessary to determine current practice patterns and to evaluate whether PCPs are following the 2012 USPSTF recommendations or alternative, more recent recommendations by others. ${ }^{19-21}$

\section{Conclusion}

PCP prostate cancer screening practice patterns as reflected in this study are generally consistent with the findings of previous focus group studies. The national survey analyzed here quantifies the magnitude of the differences reported in such focus group studies and provides insights that may be useful in hypothesis generation and the planning of future research studies.

\section{Acknowledgments and disclosure}

The authors report no conflicts of interest in this work. Battelle Centers for Public Health Research and Evaluation (Seattle, WA) performed the sample selection, survey mailing, survey completion tracking, and response rate calculation. Funding support was provided to Battelle Centers for Public Health Research and Evaluation (Seattle, WA) by Centers for Disease Control and Prevention (CDC) Contract No 2002002-00573, Task Order 04. The findings and conclusions in this report are those of the authors and do not necessarily represent the official position of the CDC.

\section{References}

1. National Program of Cancer Registries. United States cancer statistics: 1999-2007 cancer incidence and mortality data. Atlanta, GA: Centers for Disease Control and Prevention. 2012. Available from: http://apps. nccd.cdc.gov/uscs/. Accessed July 8, 2012.

2. Komaromy M, Grumbach K, Drake M, et al. The role of black and Hispanic physicians in providing health care for underserved populations. N Engl J Med. 1996;334(20):1305-1310.

3. Bach PB, Pham HH, Schrag D, Tate RC, Hargraves JL. Primary care physicians who treat blacks and whites. $N$ Engl J Med. 2004;351(6): 575-584.

4. Saha S, Shipman SA. Race-neutral versus race-conscious workforce policy to improve access to care. Health Aff (Millwood). 2008;27(1): 234-245.

5. Ross LE, Stroud LA, Rose SW, Jorgensen CM. Using telephone focus groups methodology to examine the prostate cancer screening practices of African-American primary care physicians. J Natl Med Assoc. 2006; 98(8):1296-1299.
6. Cooper CP, Merritt TL, Ross LE, John LV, Jorgensen CM. To screen or not to screen, when clinical guidelines disagree: primary care physicians' use of the PSA test. Prev Med. 2004;38(2):182-191.

7. Stroud L, Ross LE, Rose SW. Formative evaluation of the prostate cancer screening practices of African American physicians. J Natl Med Assoc. 2006;98(10):1637-1643.

8. Hall IJ, Taylor YJ, Ross LE, Richardson LC, Richards TB, Rim SH. Discussions about prostate cancer screening between US primary care physicians and their patients. J Genl Intern Med. 2011;26(10): 1098-1104.

9. Hall IJ, Ross LE, Taylor YJ, Richardson LC. Primary care physician reports of amount of time spent with male patients in prostate cancer screening discussions. J Prim Care Community Health. 2011;2(3): 192-204.

10. Werny DM, Saraiya M, Carrera J, Coughlin SS, Frank E. Learning amid controversy: prostate cancer knowledge and screening practices among US medical students. J Cancer Educ. 2008;23(2):108-113.

11. US Preventive Services Task Force. Screening for prostate cancer: recommendation and rationale. Ann Intern Med. 2002;137(11): 915-916.

12. Ransohoff DF, McNaughton Collins M, Fowler FJ. Why is prostate cancer screening so common when the evidence is so uncertain? A system without negative feedback. Am J Med. 2002;113(8):663-667.

13. Krist AH, Woolf SH, Johnson RE. How physicians approach prostate cancer screening before and after losing a lawsuit. Ann Fam Med. 2007; 5(2):120-125.

14. Asch DA, Jedrziewski MK, Christakis NA. Response rates to mail surveys published in medical journals. J Clin Epidemiol. 1997;50(10): 1129-1136.

15. Linder SK, Hawley ST, Cooper CP, Scholl LE, Jibaja-Weiss M, Volk RJ. Primary care physicians' reported use of pre-screening discussions for prostate cancer screening: a cross-sectional survey. BMC Fam Pract. 2009;10:19.

16. Royston P, Altman DG, Sauerbrei W. Dichotomizing continuous predictors in multiple regression: a bad idea. Stat Med. 2006;25(1): 127-141.

17. Montaño DE, Phillips WR. Cancer screening by primary care physicians: a comparison of rates obtained from physician self-report, patient survey, and chart audit. Am J Public Health. 1995;85(6):795-800.

18. US Preventive Services Task Force. Screening for prostate cancer: US Preventive Services Task Force recommendation statement. Ann Intern Med. 2008;149(3):185-191.

19. Moyer VA; on behalf of the US Preventive Services Task Force. Screening for Prostate Cancer: US Preventive Services Task Force Recommendation Statement. Ann Intern Med. 2012;157(2):120-134.

20. Catalona WJ, D'Amico AV, Fitzgibbons WF, et al. What the US Preventive Services Task Force missed in its prostate cancer screening recommendation. Ann Intern Med. 2012;157(2):137-138.

21. Carlsson S, Vickers AJ, Roobol M, et al. Prostate Cancer Screening: Facts, statistics, and interpretation in response to the US Preventive Services Task Force Review. J Clin Oncol. 2012;30(21):2581-2584.
International Journal of General Medicine

\section{Publish your work in this journal}

The International Journal of General Medicine is an international, peer-reviewed open-access journal that focuses on general and internal medicine, pathogenesis, epidemiology, diagnosis, monitoring and treatment protocols. The journal is characterized by the rapid reporting of reviews, original research and clinical studies across all disease areas.

\section{Dovepress}

A key focus is the elucidation of disease processes and management protocols resulting in improved outcomes for the patient.The manuscript management system is completely online and includes a very quick and fair peer-review system. Visit http://www.dovepress.com/ testimonials.php to read real quotes from published authors. 